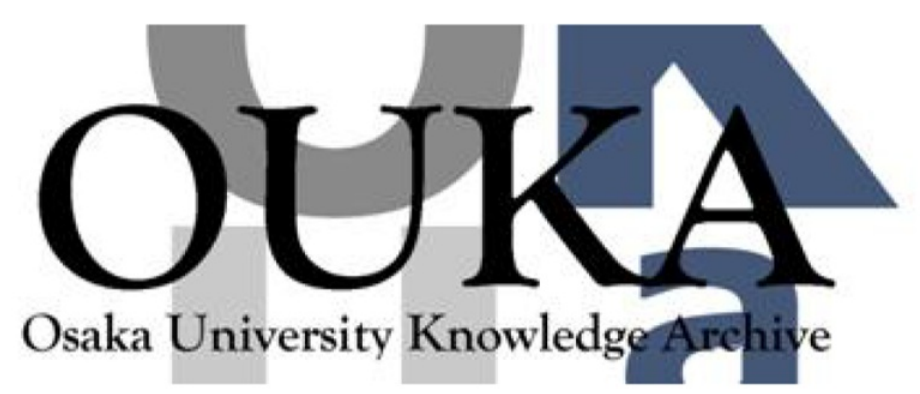

\begin{tabular}{|c|c|}
\hline Title & $\begin{array}{l}\text { Elastic, anelastic, and piezoelectric } \\
\text { coefficients of } \alpha \text {-quartz determined by } \\
\text { resonance ultrasound spectroscopy }\end{array}$ \\
\hline Author (s) & $\begin{array}{l}\text { Ogi, Hirotsugu; Ohmori, Toshinobu; Nakamura, } \\
\text { Nobutomo et al. }\end{array}$ \\
\hline Citation & $\begin{array}{l}\text { Journal of Applied Physics. 100(5) p.053511-1- } \\
\text { p. 053511-7 }\end{array}$ \\
\hline Issue Date & $2006-09-01$ \\
\hline oaire:version & VoR \\
\hline URL & https://hdl. handle. net/11094/84224 \\
\hline rights & $\begin{array}{l}\text { This article may be downloaded for personal use } \\
\text { only. Any other use requires prior permission } \\
\text { of the author and AIP Publishing. This article } \\
\text { appeared in Journal of Applied Physics, 100(5), } \\
053511 \text { (2006) and may be found at } \\
\text { https://doi.org/10.1063/1.2335684. }\end{array}$ \\
\hline Note & \\
\hline
\end{tabular}

Osaka University Knowledge Archive : OUKA

https://ir. Library. osaka-u. ac. jp/

Osaka University 


\title{
Elastic, anelastic, and piezoelectric coefficients of $\alpha$-quartz determined by resonance ultrasound spectroscopy
}

\author{
Hirotsugu Ogi, ${ }^{\text {a) }}$ Toshinobu Ohmori, Nobutomo Nakamura, and Masahiko Hirao \\ Graduate School of Engineering Science, Osaka University, Toyonaka, Osaka 560-8531, Japan
}

(Received 10 March 2006; accepted 11 July 2006; published online 8 September 2006)

\begin{abstract}
All independent components of the elastic constants, internal friction, and piezoelectric coefficients of synthetic $\alpha$-quartz have been simultaneously determined by resonance ultrasound spectroscopy coupled with laser-Doppler interferometry. Seventeen crystals obtained from $Z$ and $X$ regions with various infrared-absorption values were used; for each crystal, a complete set of the coefficients was determined using 72 resonance frequencies, which were measured by a needle-transducer tripod in a vacuum at a constant temperature with frequency-error limit of $0.001 \%$. The infrared-absorption value and grown region did not strongly affect the material coefficients. Among the eight (elastic plus piezoelectric) coefficients, piezoelectric coefficients were significantly different from previously reported values. The six independent internal-friction components showed a positive correlation with the temperature derivatives of the corresponding elastic constants, indicating that the dominant damping mechanism was the phonon-phonon scattering. Our values at $30^{\circ} \mathrm{C}$ are $C_{11}=86.76 \mathrm{GPa}, \quad C_{12}=6.868 \mathrm{GPa}, \quad C_{13}=11.85 \mathrm{GPa}, C_{14}=-18.02 \mathrm{GPa}, C_{33}=105.5 \mathrm{GPa}, C_{44}$ $=58.14 \mathrm{GPa}$, and $e_{11}=0.151 \mathrm{C} / \mathrm{m}^{2}$, and $e_{14}=-0.061 \mathrm{C} / \mathrm{m}^{2}$. (C) 2006 American Institute of Physics.
\end{abstract}

[DOI: $10.1063 / 1.2335684]$

\section{INTRODUCTION}

$\alpha$-quartz has greatly benefited humans with its favorable piezoelectricity. It possesses 32-point-group symmetry, showing six independent elastic constants $C_{i j}^{E}$, two independent piezoelectric coefficients $e_{i j}$, and two dielectric constants $\epsilon_{i j}^{S}{ }^{1}$ Besides, six internal frictions $Q_{i j}^{-1}$ accompany the elastic constants. ${ }^{2,3}$ These material constants are essential for designing acoustic devices and have been reported by several researchers: Bechmann, ${ }^{4}$ Bechmann et al. ${ }^{5}$ and Koga et al. ${ }^{6}$ used the plate-thickness resonance method; James ${ }^{7}$ determined the elastic constants by the pulse-echo method assuming the piezoelectric constants given by Cook and Weissler; ${ }^{8}$ and Kushibiki et al. ${ }^{9}$ determined all the material constants except for internal friction by the pulse-echo method. These previous studies, however, required many independent measurements on many crystals in many orientations to determine a complete set of coefficients through solving labyrinthine simultaneous equations. Various errors could occur, being associated with the use of different specimens, crystal misorientations, resonance-frequency shifts by attaching electrodes and coupling materials, ambiguous electric boundary conditions at the interfaces between the specimen and the coupling material, and so on. The complete internal-friction tensor $Q_{i j}^{-1}$ of quartz has never been reported regardless of numerous studies on the elastic and piezoelectric properties.

Here, we intend to determine the independent elastic, anelastic, and piezoelectric coefficients of $\alpha$-quartz by resonance ultrasound spectroscopy (RUS). All the $C_{i j}^{E}$ and the combinations of $e_{i j}$ and $\epsilon_{i j}^{S}$ affect mechanical free-vibration resonance frequencies of a piezoelectric material with different contributions, and we can, in principle, determine in-

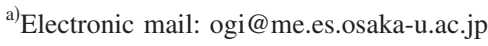

versely all the coefficients by measuring many resonance frequencies. This approach was suggested by Ohno ${ }^{10}$ and Dunn et al. ${ }^{11}$ but was not actualized because of the small contributions of $e_{i j}$ and mode-identification difficulties. We recently developed the needle-transducer tripod to measure the resonance frequencies of small specimens with sufficiently high accuracy to determine $e_{i j}$ as well as $C_{i j}^{E}$. Furthermore, we used laser-Doppler interferometry (LDI) to identify the vibrational modes unambiguously. ${ }^{12}$ We call this method the RUS/LDI method, and we applied it to study lithium niobate, ${ }^{13}$ langasite, ${ }^{3}$ and paratellurite. ${ }^{14}$ This task, however, becomes much more difficult for $\alpha$-quartz because of much smaller magnitudes of the piezoelectric coefficients; their normalized sensitivities to the resonance frequencies are between 0.001 and 0.05 . Therefore, we must measure and calculate the resonance frequencies very accurately. Even the ambient-pressure effect should be eliminated. Internalfriction measurement should also be done under vacuum condition to minimize the leakage of the vibrational energy into the air.

Here, we measure the resonance frequencies in a vacuum at a constant temperature and determine all the $C_{i j}^{E}$, $e_{i j}$, and $Q_{i j}^{-1}$ of synthetic quartz crystals obtained from various ingots with different infrared-absorption coefficients and from different regions. In total, 17 crystals were used and these material constants were determined individually.

\section{SPECIMENS}

Material constants of $\alpha$-quartz are written in contracted notation as 
TABLE I. Infrared-absorption value $\alpha\left(\mathrm{cm}^{-1}\right)$, elastic constants $C_{i j}^{E}(\mathrm{GPa})$, piezoelectric coefficients $e_{i j}\left(\mathrm{C} / \mathrm{m}^{2}\right)$, and mass density $\rho\left(\mathrm{kg} / \mathrm{m}^{3}\right)$ of $\alpha$-quartz crystals at $30^{\circ} \mathrm{C}$. The rms errors between measured and calculated resonance frequencies after convergence of the inverse calculation are also shown.

\begin{tabular}{|c|c|c|c|c|c|c|c|c|c|c|c|c|}
\hline$\alpha$ & Specimen & $C_{11}$ & $C_{12}$ & $C_{13}$ & $C_{14}$ & $C_{33}$ & $C_{44}$ & $C_{66}{ }^{\mathrm{a}}$ & $e_{11}$ & $e_{14}$ & $\rho$ & rms error $(\%)$ \\
\hline \multirow[t]{3}{*}{0.024} & QZ1-1 (1) & 86.70 & 6.808 & 11.75 & -18.06 & 105.42 & 58.22 & 39.94 & 0.150 & -0.041 & 2643.51 & 0.068 \\
\hline & QZ1-2 (2) & 86.73 & 6.913 & 11.82 & -18.03 & 105.45 & 58.21 & 39.91 & 0.155 & -0.050 & 2645.58 & 0.036 \\
\hline & QZ1-3 (3) & 86.86 & 6.896 & 11.85 & -18.07 & 105.54 & 58.28 & 39.98 & 0.151 & -0.051 & 2645.31 & 0.055 \\
\hline \multirow[t]{5}{*}{0.049} & QZ2-1 (4) & 86.72 & 6.925 & 11.87 & -17.92 & 105.11 & 58.07 & 39.9 & 0.145 & -0.077 & 2642.21 & 0.061 \\
\hline & QZ2-2 (5) & 86.70 & 6.917 & 11.89 & -17.91 & 105.23 & 58.09 & 39.89 & 0.147 & -0.082 & 2645.02 & 0.063 \\
\hline & QZ2-3 (6) & 86.69 & 6.935 & 11.81 & -17.98 & 105.33 & 58.24 & 39.88 & 0.155 & -0.062 & 2644.13 & 0.052 \\
\hline & QZ2-4 (7) & 86.76 & 6.872 & 11.94 & -18.01 & 105.43 & 58.2 & 39.94 & 0.139 & -0.057 & 2646.49 & 0.061 \\
\hline & QZ2-5 (8) & 86.71 & 6.897 & 11.89 & -18.01 & 105.48 & 58.25 & 39.91 & 0.150 & -0.050 & 2644.86 & 0.057 \\
\hline \multirow[t]{3}{*}{0.068} & QZ3-1 (9) & 86.95 & 6.933 & 11.9 & -18.06 & 105.62 & 58.09 & 40.01 & 0.155 & -0.066 & 2643.78 & 0.082 \\
\hline & QZ3-2 (10) & 86.79 & 6.900 & 11.82 & -18.04 & 105.54 & 58.11 & 39.94 & 0.159 & -0.057 & 2644.81 & 0.035 \\
\hline & QZ3-3 (11) & 86.86 & 6.801 & 11.92 & -18.12 & 105.6 & 58.06 & 40.03 & 0.151 & -0.053 & 2645.55 & 0.070 \\
\hline \multirow[t]{4}{*}{0.12} & QZ4-1 (12) & 86.69 & 6.956 & 11.79 & -17.98 & 105.36 & 58.02 & 39.87 & 0.159 & -0.068 & 2641.70 & 0.066 \\
\hline & QZ4-2 (13) & 86.75 & 6.918 & 11.67 & -17.99 & 105.46 & 58.03 & 39.92 & 0.158 & -0.071 & 2644.90 & 0.045 \\
\hline & QZ4-3 (14) & 86.80 & 6.851 & 11.82 & -18.08 & 105.54 & 58.12 & 39.97 & 0.158 & -0.051 & 2644.66 & 0.029 \\
\hline & QZ4-4 (15) & 86.86 & 6.665 & 11.84 & -18.07 & 105.5 & 58.05 & 40.1 & 0.133 & -0.061 & 2646.25 & 0.061 \\
\hline \multirow[t]{4}{*}{0.12} & QX-1 (16) & 86.66 & 6.785 & 11.93 & -18.01 & 105.56 & 58.16 & 39.94 & 0.148 & -0.065 & 2645.92 & 0.067 \\
\hline & QX-2 (17) & 86.74 & 6.784 & 11.95 & -18.01 & 105.63 & 58.19 & 39.98 & 0.147 & -0.067 & 2644.82 & 0.074 \\
\hline & Average & 86.76 & 6.868 & 11.85 & -18.02 & 105.46 & 58.14 & 39.95 & 0.151 & -0.061 & 2644.67 & 0.058 \\
\hline & $\mathrm{SD}(\%)$ & 0.09 & 1.11 & 0.62 & 0.30 & 0.13 & 0.14 & 0.15 & 4.75 & 17.8 & 0.049 & $\ldots$ \\
\hline
\end{tabular}

${ }^{\mathrm{a}} C_{66}=\left(C_{11}-C_{12}\right) / 2$.

$$
\begin{aligned}
& {\left[C_{i j}\right]=\left[\begin{array}{cccccc}
C_{11} & C_{12} & C_{13} & C_{14} & 0 & 0 \\
C_{12} & C_{11} & C_{13} & -C_{14} & 0 & 0 \\
C_{13} & C_{13} & C_{33} & 0 & 0 & 0 \\
C_{14} & -C_{14} & 0 & C_{44} & 0 & 0 \\
0 & 0 & 0 & 0 & C_{44} & C_{14} \\
0 & 0 & 0 & 0 & C_{14} & C_{66}
\end{array}\right],} \\
& {\left[e_{i j}\right]=\left[\begin{array}{cccccc}
e_{11} & -e_{11} & 0 & e_{14} & 0 & 0 \\
0 & 0 & 0 & 0 & -e_{14} & -e_{11} \\
0 & 0 & 0 & 0 & 0 & 0
\end{array}\right] \text {, }} \\
& {\left[\epsilon_{i j}^{S}\right]=\left[\begin{array}{ccc}
\epsilon_{11}^{S} & 0 & 0 \\
0 & \epsilon_{11}^{S} & 0 \\
0 & 0 & \epsilon_{33}^{S}
\end{array}\right] .}
\end{aligned}
$$

We omit the superscript $E$ for $C_{i j}$ for simplicity. All the coefficients contribute to the free-vibration resonance frequencies, but $e_{i j}$ and $\epsilon_{i j}^{S}$ cannot be determined separately because their combinations affect the mechanical vibrations. We then used the averaged values given by Bechmann, ${ }^{4}$ James, ${ }^{7}$ and Kushibiki et al. ${ }^{9}$ for the dielectric constants as $\epsilon_{11}^{S} / \epsilon_{0}$ $=4.424$ and $\epsilon_{33}^{S} / \epsilon_{0}=4.632$, where $\epsilon_{0}$ denotes the vacuum permittivity. (The dielectric constants accurately obtained from the low-frequency capacitance measurement by several researchers agree well.)

Seventeen specimens were cut into rectangular parallelepipeds from different quartz ingots synthesized by hydrothermal crystallization method. Their infrared-absorption values ( $\alpha$ values) at $3585 \mathrm{~cm}^{-1}$, corresponding to a $\mathrm{OH}$ absorption, ${ }^{15}$ ranged between 0.024 and $0.12 \mathrm{~cm}^{-1}$, which has been correlated with the $Q$ value of quartz oscillators. ${ }^{16}$ The specimens were mainly obtained from the $-Z$ region, but two specimens were from the $+X$ region, where the dislocation density is much higher, ${ }^{17}$ for investigating the effect of dislocations on the material constants. (The definition of the grown region, such as $+X$, is shown in detail in Ref. 17.) We classify the specimens into five groups; QZ1, QZ2, QZ3, QZ4, and QX, reflecting the infrared-absorption value and the grown region (see Table I). Dimensions of the specimens were about $4.8,5.2$, and $5.8 \mathrm{~mm}$ along the crystallographic $X, Y$, and $Z$ axes, respectively, which were the averages of ten measurements using a micrometer with the absolute accuracy of $1 \mu \mathrm{m}$. Thus, the error from the dimension measurement is expected to be less than $0.02 \%$. By Laue x-ray diffraction, we confirmed the crystal orientation within $1^{\circ}$. Specimen mass was measured by a microbalance with the accuracy of $0.002 \%$ and the mass density was determined from the mass and dimensions.

\section{METHODS}

Details of the RUS/LDI method appear elsewhere. ${ }^{3,12,13}$ In this study, all the components were placed in a vacuum chamber $(\sim 1 \mathrm{~Pa})$ to avoid the effect of the ambient pressure on the resonance frequencies and the leakage of the vibrational energy into air. The specimen was put on the needletransducer tripod, consisting of two needle-type transducers for generation and detection and one needle-thermocouple support, which touched the specimen and measured the specimen temperature. No external force was applied to the specimen except for gravity. The resonance frequencies were measured at $30{ }^{\circ} \mathrm{C}$ three times for each specimen to see the 


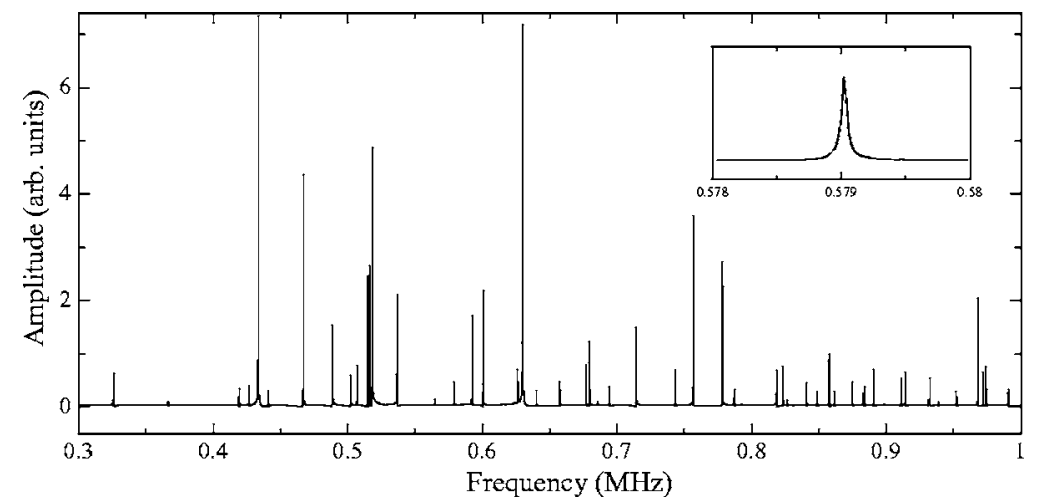

FIG. 1. Resonance spectrum observed for QZ1-1 in a vacuum. The inset shows resonance-peak shape.

reproducibility; after measuring the resonance frequencies, we took the specimen out of the vacuum chamber, replaced it on the tripod, and again measured the frequencies in vacuum. Differences of the resonance frequencies among the three measurements were smaller than $0.001 \%$ regardless of the different contact points between the specimen and the needles. Thus, high reproducibility was confirmed. Figure 1 shows an example of the measured resonance spectrum.

Inverse calculation was performed by Lagrangian minimization with a Rayleigh-Ritz approach. ${ }^{3,10,14}$ The displacements and electric potential were expanded into linear combinations of basis factions $\phi_{k}$ composed of Legendre functions:

$$
\begin{aligned}
\phi_{k}= & \frac{1}{\sqrt{L_{1} L_{2} L_{3}}} \sqrt{\frac{2 l+1}{2}} \sqrt{\frac{2 m+1}{2}} \sqrt{\frac{2 n+1}{2}} \\
& \times P_{l}\left(\frac{x_{1}}{L_{1}}\right) P_{m}\left(\frac{x_{2}}{L_{2}}\right) P_{n}\left(\frac{x_{3}}{L_{3}}\right) .
\end{aligned}
$$

Hence $x_{1}, x_{2}$, and $x_{3}$ denote Cartesian coordinates along the $X, Y$, and $Z$ crystallographic axes, respectively. $L_{i}$ denotes the edge length along the $x_{i}$ axis of the rectangularparallelepiped crystal. $l, m$, and $n$ denote the order numbers of the Legendre polynomial. Poor contributions of the piezoelectric coefficients to the resonance frequencies forced us to involve higher-order Legendre polynomials than did previous studies. Figure 2 shows differences of the calculated resonance frequencies from those calculated using many Legendre polynomials up to $N=26$, where $N=l+m+n$. The case of $N=26$ involves a sufficiently large number of basis functions, and it provides resonance frequencies close to the true values. Thus, Fig. 2 indicates that we can calculate the resonance frequencies of the first 80 modes with errors less than $0.0034 \%$ when we use Legendre functions up to $N$ $=18$. The total number of basis functions in this case reaches near 15 000, implying a very long calculation time. However, because the free vibrations of an oriented rectangularparallelepiped $\alpha$-quartz crystal fall into four vibrational groups labeled as $A_{g}, B_{g}, A_{u}$, and $B_{u}$, according to the deformation symmetry, ${ }^{10}$ this number decreases to about 3500 for each group, requiring much shorter calculation time (a few minutes with a conventional desktop computer).

We have to pair up a measured frequency with a calculated frequency in the inverse calculation. Because the vibrational modes of the calculated frequencies are exactly known, we must identify observed frequencies. This task, mode identification, is essential in RUS studies and mode misidentification is fatal for determining weakly contributing piezoelectric coefficients. We achieved mode identification by the LDI method: after completing all the frequency and the internal-friction measurements, we deposited a $100 \mathrm{~nm}$ aluminum thin film on the $Z$ face of the crystal and scanned the resonating surface with a $\mathrm{He}-\mathrm{Ne}$ laser beam, measuring the Doppler frequency shift of the laser beam, which was proportional to the particle velocity or to the displacement amplitude in harmonic oscillation. The diameter of the laser spot was about $50 \mu \mathrm{m}$. Then, measured out-of-planedisplacement distributions were compared with those calculated. Figure 3 shows four examples of comparison between the measured and calculated displacement distributions, showing excellent agreement. Such a vibrational-mode figure is uniquely determined for an individual mode, like a fingerprint. Thus, comparison between the measured and calculated displacement distributions leads us to unambiguous mode identification. We identified 72 resonance modes, whose frequencies were used to determine the eight coefficients.

Internal friction $Q_{i j}^{-1}$ can be defined as the ratio of imaginary to real parts of the complex elastic stiffness. ${ }^{2}$

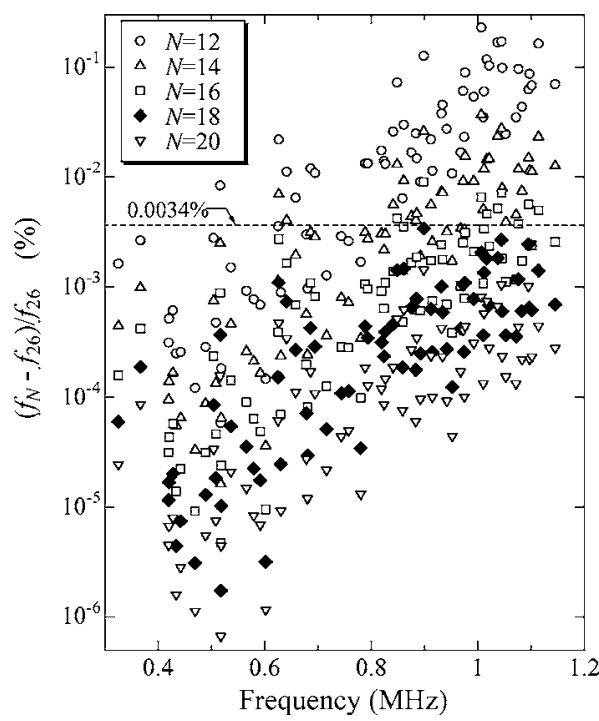

FIG. 2. Normalized differences of resonance frequencies calculated with various $N(=l+m+n)$ values from those with sufficiently large number of Legendre basis functions with $N=26$. Specimen was QZ1-1. 

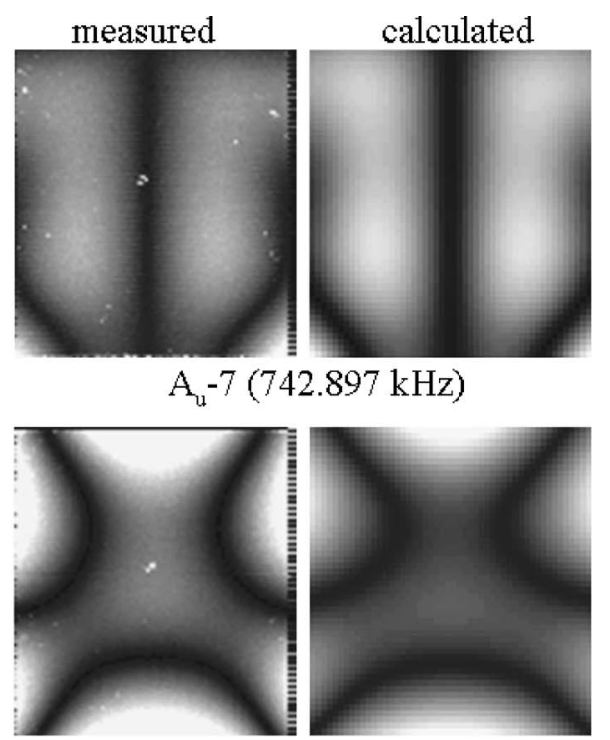

$\mathrm{A}_{\mathrm{g}}-10(817.590 \mathrm{kHz})$

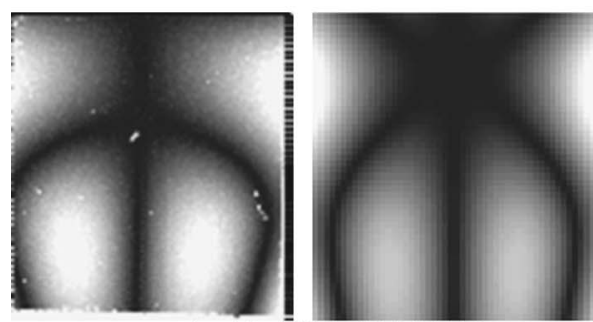

$\mathrm{B}_{\mathrm{g}}-10(882.620 \mathrm{kHz})$

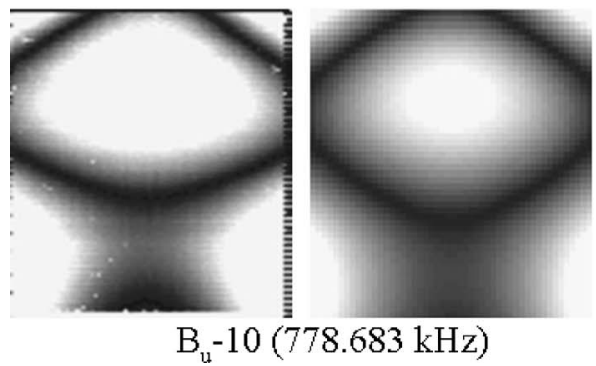

FIG. 3. Measured (left) and calculated (right) distributions of the out-ofplane displacement on the $Z$ surface of QZ1-1. The bright regions are antinode regions and the dark lines represent nodal lines. The maximum amplitude was about $0.8 \mathrm{~nm}$. These figures provide unambiguous vibration-mode identification.

$$
\widetilde{C}_{i j}=C_{i j}\left(1+j Q_{i j}^{-1}\right) .
$$

There are six independent internal frictions in $\alpha$-quartz. The internal-friction tensor $Q_{i j}^{-1}$ permits us to predict unmeasurable mechanical loss of any ultrasonic mode and helps select less lossy propagation and polarization directions in designing acoustic devices. The independent components are inversely determined from the $Q^{-1}$ values of many vibrational modes using the calculated contribution of each internalfriction component to the observed value. ${ }^{3}$ The $Q^{-1}$ value of each resonance peak was determined from its half-maximum peak width. Unlike the elastic constants and piezoelectric coefficients, the internal-friction measurement is easily affected by the contact conditions at the specimen/transducer interfaces. We then measured the $Q^{-1}$ values of individual

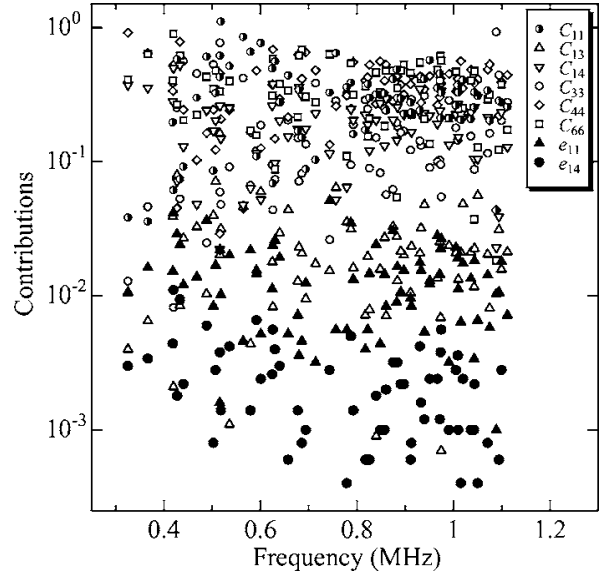

FIG. 4. Normalized contributions of eight coefficients $|p / f(\partial f / \partial p)|$ to resonance frequencies calculated for the crystal QZ1-1.

peaks three times for each specimen and averaged the results in each specimen group.

\section{RESULTS AND DISCUSSION}

Table I shows the determined coefficients for all specimens. We define $|p / f(\partial f / \partial p)|$ as the contribution of a particular coefficient $p$ (one of $C_{i j}$ and $e_{i j}$ ) to a resonance frequency $f$. Figure 4 shows the contributions of the eight coefficients calculated by the Rayleigh-Ritz method. The contributions of $e_{i j}$ are between $0.1 \%$ and 5\%, indicating that we must measure and calculate the resonance frequencies and measure dimensions and mass density with errors one or two orders of magnitude smaller than the contributions. This demand was also required for the conventional pulse-echo method, but a velocity measurement with $0.01 \%$ error limit would be difficult because of uncertain electric boundary conditions at the coupling-material/specimen interface, nontrivial correction for diffraction, wave form distortion caused by the internal reflections within the coupling material, and so on. On the other hand, the resonance frequencies are unambiguously measured with the error limit of $0.001 \%$ by the RU/LDI measurement in a vacuum without using any coupling materials and applied forces. They are also accurately calculated with errors smaller than $0.0034 \%$ (Fig. 2). Mass of the specimen was measured by a microbalance with an accuracy of $0.002 \%$. The maximum error is then involved in dimensions, which is about $0.02 \%$, and minimized by averaging the resultant coefficients of many specimens.

Figure 5 shows differences of the measured resonance frequencies and those calculated involving (i) both $e_{11}$ and $e_{14}$, (ii) only $e_{14}$, and (iii) only $e_{11}$. The rms difference between measurements and calculations becomes minimum $(\sim 0.06 \%)$ when both coefficients are involved, demonstrating that the piezoelectric coefficients sufficiently affect the resonance frequencies. Furthermore, the previous studies used several different specimens to determine a set of coefficients, but we determine all the coefficients simultaneously from only one small specimen using a much larger number of measurements. (Use of 72 frequencies corresponds to 72 


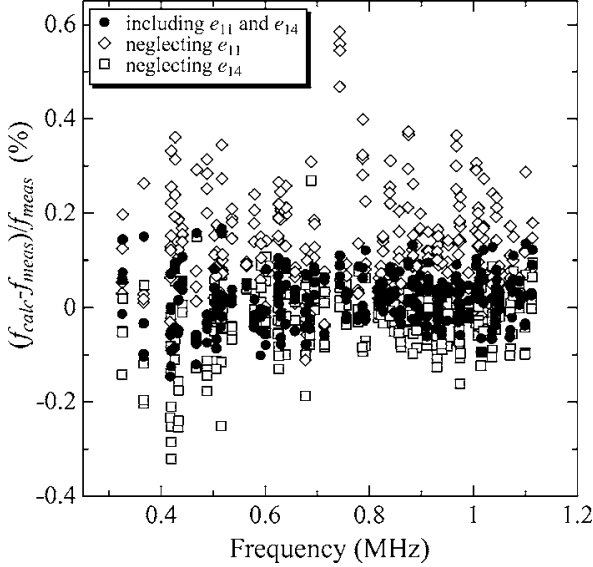

FIG. 5. Comparison between measured resonance frequencies and calculated ones including $e_{11}$ and $e_{14}$ and neglecting $e_{11}$ and $e_{14}$. Results for QZ1-1, QZ2-1, QZ3-1, and QZ4-1 are shown together.

measurements in different directions with various polarizations in the pulse-echo method.) Therefore, the accuracy in the present study is expected to be higher.

Figures 6 and 7 compare our results with previous reports. Sorokin et al. ${ }^{18}$ suggested that $C_{66}$ was affected by the crystal grade ( $\alpha$ value), but we observe no remarkable dependences of the coefficients on the crystal grade and the grown region. We then averaged all the results of the 17 specimens to provide the eight (elastic plus piezoelectric) coefficients of $\alpha$-quartz determined by the RUS/LDI method,

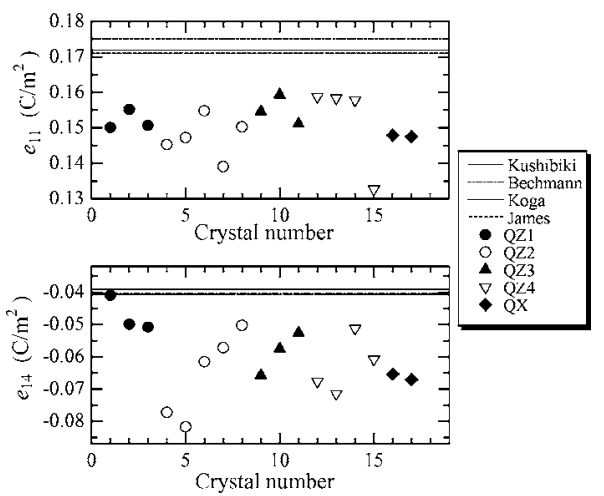

FIG. 7. Piezoelectric coefficients determined by the RUS/LDI method. Previous reports are shown by lines for comparison.

which are shown in Table II together with those reported previously. We note that previous values agree well with each other even for the small contributing $e_{14}$. This agreement is most surprising considering the ambiguity associated with the contacting measurements. Our values agree with the previous values within the error limits except for $C_{33}$ and the two piezoelectric coefficients; our $C_{33}$ is smaller than the previous values by $\sim 0.3 \%$, our $e_{11}$ is smaller by $\sim 15 \%$, and our $e_{14}$ is larger in magnitude by $\sim 35 \%$. The temperature dependence of $C_{33}$ partially explains this discrepancy; using the temperature coefficient of $-1.93 \times 10^{-4} \mathrm{~K}^{-1}$ for $C_{33}$, ${ }^{9}$ we find that $C_{33}$ decreases by $0.2 \%$ with a $10 \mathrm{~K}$ temperature increase. However, the temperature dependence of $e_{i j}$ is
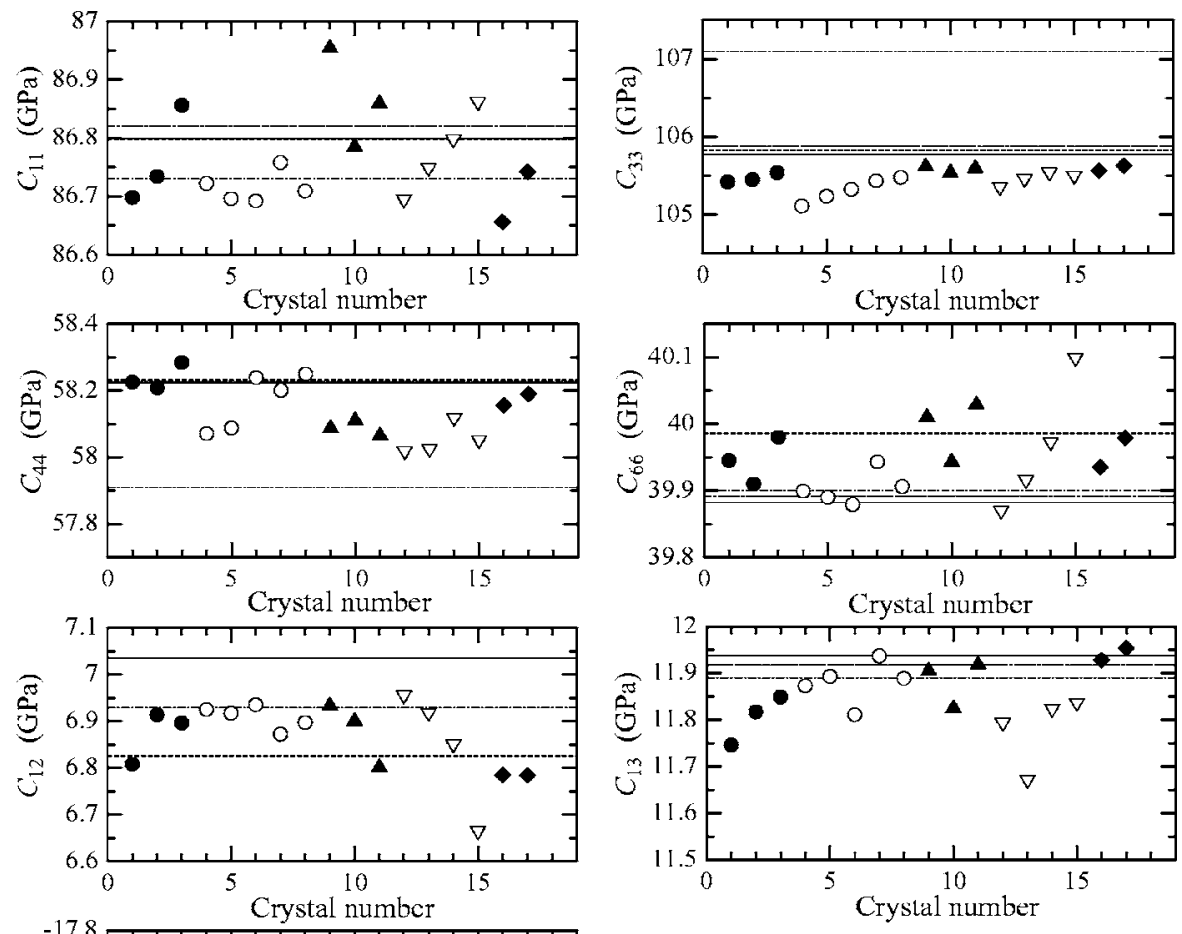

FIG. 6. Elastic constants determined by the RUS/LDI method. Previous reports are shown by lines for comparison.
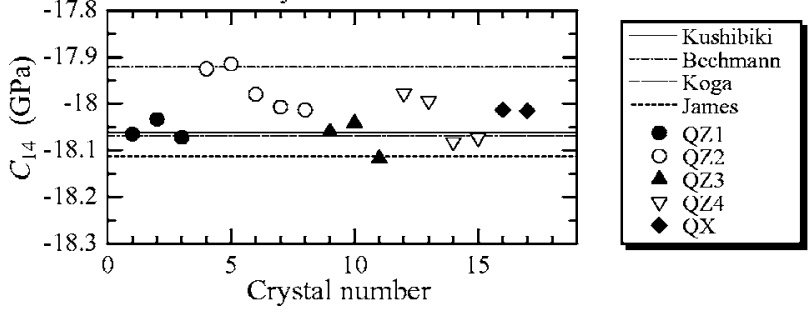
TABLE II. Elastic constants $C_{i j}^{E}(\mathrm{GPa})$ and piezoelectric coefficients $e_{i j}\left(\mathrm{C} / \mathrm{m}^{2}\right)$ of $\alpha$-quartz.

\begin{tabular}{lccccc}
\hline \hline & $\begin{array}{c}\text { Present } \\
\left(30^{\circ} \mathrm{C}\right)\end{array}$ & $\begin{array}{c}\text { Kushibiki } \text { et al. (Ref. 9) } \\
\left(23^{\circ} \mathrm{C}\right)\end{array}$ & $\begin{array}{c}\text { James (Ref. 7) } \\
\left(25^{\circ} \mathrm{C}\right)\end{array}$ & $\begin{array}{c}\text { Koga et al. (Ref. 6) } \\
\left(20^{\circ} \mathrm{C}\right)\end{array}$ & $\begin{array}{c}\text { Bechmann (Ref. 4) } \\
\left(20^{\circ} \mathrm{C}\right)\end{array}$ \\
\hline$C_{11}$ & 86.76 & 86.80 & 86.79 & 86.83 & 86.74 \\
$C_{12}$ & 6.868 & 7.036 & 6.790 & 7.090 & 6.99 \\
$C_{13}$ & 11.85 & 11.94 & 12.01 & 11.93 & 11.91 \\
$C_{14}$ & -18.02 & -18.06 & -18.12 & -18.06 & -17.91 \\
$C_{33}$ & 105.46 & 105.78 & 105.79 & 105.94 & 107.2 \\
$C_{44}$ & 58.14 & 58.22 & 58.21 & 58.26 & 57.94 \\
$C_{66}{ }^{a}$ & 39.95 & 39.88 & 40.00 & 39.87 & 39.88 \\
$e_{11}$ & 0.151 & 0.172 & 0.171 & 0.175 & 0.171 \\
$e_{14}$ & -0.061 & -0.039 & -0.041 & -0.041 & -0.041 \\
\hline${ }^{{ }^{\mathrm{a}} C_{66}=\left(C_{11}-C_{12}\right) / 2 .}$ & &
\end{tabular}

much smaller. Therefore, the discrepancies of our piezoelectric coefficients from those reported are significant.

Internal-friction values measured in a vacuum were smaller than those measured in ambient pressure by factors of 1.2-2.8, indicating the necessity of the internal-friction measurement in a vacuum. Figure 8 shows the diagonal internal frictions determined inversely, which are of the order of $10^{-5}$. Heyliger et al. ${ }^{19}$ measured internal friction of a natural quartz by the conventional RUS method, giving $Q^{-1}$ values of about $5 \times 10^{-4}$. They noted that this value was larger than that of a high-quality quartz $\left(\sim 1.0 \times 10^{-5}\right)$, and they suggested that this discrepancy was caused by the higher dislocation density in their specimen. However, our result did not show significant dependence on the grown region. Dislocation density is much larger in the $X$ region of a synthetic quartz, ${ }^{17}$ but lower internal friction of the QX specimens disputes the dislocation principal damping mechanism. Furthermore, the $\alpha$ value affected internal friction very little. The $\alpha$ value indicates the impurity concentration and has been correlated with the $Q$ value of a quartz oscillator. ${ }^{16}$ The result in Fig. 8 indicates that such a damping mechanism will be insignificant for frequencies below $1 \mathrm{MHz}$ used here.

We therefore consider phonon-phonon interactions as the major damping mechanism in the frequency region used here. Acoustic vibrations disturb the equilibrium state of thermal phonons, and scattered phonons tend to equilibrate through interactions with low-frequency acoustic phonons and thermal-mode phonons. This process is irreversible and

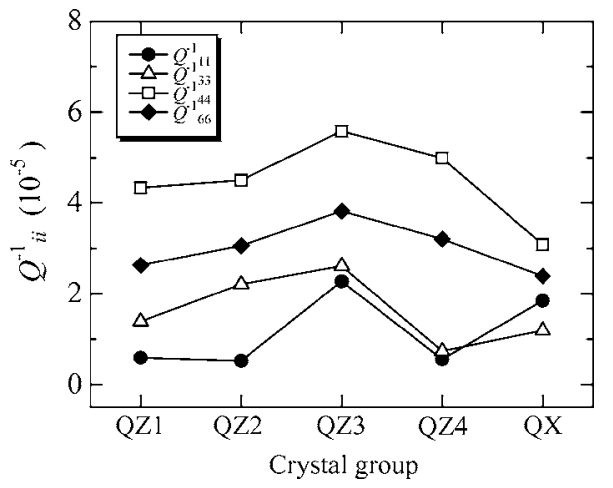

FIG. 8. Diagonal components of the internal-friction tensor. Note that two shear components exceed two longitudinal components. causes energy loss. The energy loss is determined by lattice anharmonicity and relates closely with a dimensionless constant, the Grüneisen parameter $\gamma\left(\right.$ Akheiser mechanism ${ }^{20}$ ). In a low-frequency region, the loss is proportional to $\gamma^{2} / \rho v^{2}$, where $\rho$ and $v$ denote the mass density and the sound velocity, respectively. ${ }^{21}$ We assume that this relationship applies to individual modes, that is,

$$
Q_{i j}^{-1} \propto \frac{\gamma_{i j}^{2}}{C_{i j}},
$$

where $\gamma_{i j}$ denotes the Grüneisen parameter of the vibrational mode governed by $C_{i j}$. The mode Grüneisen parameter is also related with the temperature derivatives of the corresponding elastic constants. Ledbetter ${ }^{22}$ derived a formulation connecting the bulk-modulus temperature derivative with $\gamma$. We here assume that his result holds between $C_{i j}$ and $\gamma_{i j}$ :

$$
\frac{1}{C_{i j}}\left(\frac{\partial C_{i j}}{\partial T}\right)=-\frac{3 k \gamma_{i j}\left(\gamma_{i j}+1\right)}{C_{i j} V_{a}} .
$$

Here, $V_{a}$ denotes the atomic volume and $k$ the Boltzmann constant. Therefore, the magnitude of the temperature derivative of $C_{i j}$ is expected to show a positive correlation with corresponding internal friction $Q_{i j}^{-1}$ provided that the phononphonon interaction is the dominant damping mechanism. Figure 9 compares $Q_{i j}^{-1}$ determined here and the temperature derivatives of $C_{i j}$ reported by Bechmann et al. ${ }^{5} Q_{i j}^{-1}$ corre-

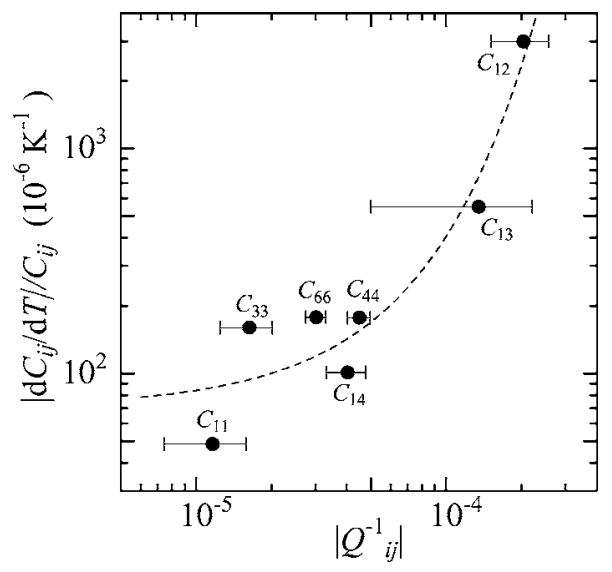

FIG. 9. Internal-friction components vs temperature derivatives of the elastic constants. 
lates with the temperature derivative of $C_{i j}$, implying a significant phonon-phonon scattering loss. Such a positive correlation was also observed in an $\alpha-\mathrm{TeO}_{2}$ monocrystal, ${ }^{14}$ and this correlation may be commonly observed in piezoelectric materials.

However, we have to point out that the internal-friction value estimated by the theory for the Akheiser mechanism $\left(\sim 10^{-8}\right)$ is much smaller than the measured values at room temperature $\left(\sim 10^{-5}\right)$. (We estimated the theoretical value using reported specific heat and thermal conductivity.) One may attribute this discrepancy to dislocation-related mechanisms, such as damping caused by scattering of phonons by moving dislocations. ${ }^{23}$ However, the small $X$-region internal friction poses a negative view for a dislocation-related mechanism. Therefore, identification of the dominant damping mechanisms remains, and we may need an improved theory for the phonon-scattering loss.

\section{CONCLUSION}

The RUS/LDI method was used for determining all the elastic, anelastic, and piezoelectric coefficients of 17 $\alpha$-quartz crystals. To overcome the weak sensitivity of the piezoelectric coefficients to the resonance frequencies, the measurements were done in a vacuum at constant temperature. Seventy-two resonance peaks were identified by laserDoppler interferometry and used to determine inversely the eight coefficients for each specimen. The crystal grade and grown region did not affect them significantly. Our coefficients agreed with those reported previously except for the piezoelectric coefficients; $e_{11}$ is smaller by $15 \%$ and $e_{11}$ is larger by $35 \%$ than the existing values. Considering the accuracy for the frequency measurement, we consider that our values are more reliable. Internal friction showed a positive correlation with the temperature derivative of the corre- sponding elastic constant, indicating that the damping principally arises from phonon-phonon scattering, although the internal-friction magnitude does not agree with the theory.

${ }^{1}$ J. F. Nye, Physical Properties of Crystals (Oxford University Press, Oxford, 1957), p. 299

${ }^{2}$ H. Ledbetter, C. Fortunko, and P. Heyliger, J. Mater. Res. 10, 1352 (1995).

${ }^{3}$ H. Ogi, N. Nakamura, K. Sato, M. Hirao, and S. Uda, IEEE Trans. Ultrason. Ferroelectr. Freq. Control 50, 553 (2003).

${ }^{4}$ R. Bechmann, Phys. Rev. 110, 1060 (1958).

${ }^{5}$ R. Bechmann, A. D. Ballato, and T. J. Lukaszek, Proc. IRE 50, 1812 (1962).

${ }^{6}$ I. Koga, M. Aruga, and Y. Yoshinaka, Phys. Rev. 109, 1467 (1958).

${ }^{7}$ B. J. James, Proceedings of the 42nd Annual Frequency Control Symposium, (IEEE, Baltimore, 1988), p. 146.

${ }^{8}$ R. K. Cook and P. G. Weissler, Phys. Rev. 80, 712 (1950).

${ }^{9}$ J. Kushibiki, I. Takanaga, and S. Nishiyama, IEEE Trans. Ultrason. Ferroelectr. Freq. Control 49, 125 (2002).

${ }^{10}$ I. Ohno, Phys. Chem. Miner. 17, 371 (1990).

${ }^{11}$ M. Dunn, H. Ledbetter, and P. Heyliger, Engineering Mechanics (ASME, Washington, 1995), p. 758.

${ }^{12}$ H. Ogi, K. Sato, T. Asada, and M. Hirao, J. Acoust. Soc. Am. 112, 2553 (2002).

${ }^{13}$ H. Ogi, H. Ledbetter, Y. Kawasaki, and K. Sato, J. Appl. Phys. 92, 2451 (2002).

${ }^{14}$ H. Ogi, M. Fukunaga, M. Hirao, and H. Ledbetter, Phys. Rev. B 69, 024104 (2004).

${ }^{15}$ J. C. Brice and A. M. Cole, J. Phys. D 12, 459 (1979).

${ }^{16}$ D. M. Dodd and D. B. Fraser, J. Phys. Chem. Solids 26, 673 (1965).

${ }^{17}$ A. Shinohara, M. C. Iano, and C. K. Suzuki, IEEE Trans. Ultrason. Ferroelectr. Freq. Control 47, 1199 (2000).

${ }^{18}$ B. P. Sorokin, D. A. Glushkov, P. P. Turchin, S. V. Michailyuta, and K. S. Aleksandrov, Proceedings of the 2000 IEEE/EIA International Frequency Control Symposium and Exhibition (IEEE, New York, 2000), p. 410.

${ }^{19}$ P. Heyliger, H. Ledbetter, and S. Kim, J. Acoust. Soc. Am. 114, 644 (2003).

${ }^{20}$ A. Akhieser, J. Phys. (USSR) 1, 277 (1939).

${ }^{21}$ W. Mason, in Physical Acoustics, edited by W. Mason (Academic, New York, 1965), Vol. IIIB, p. 254.

${ }^{22}$ H. Ledbetter, Phys. Status Solidi B 181, 81 (1994).

${ }^{23}$ G. Leibfried, Z. Phys. 127, 344 (1950). 